\title{
Usos do arduino e mit app inventor na comunicação digital: revisão sistemática
}

\author{
Uses of arduino and mit app inventor in digital communication: systematic review \\ Usos de arduino y mit app inventor en la comunicación digital: revisión sistemática
}

Recebido: 03/02/2022 | Revisado: 08/02/2022 | Aceito: 19/02/2022 | Publicado: 28/02/2022

\author{
Juliano Moreto Massoca \\ ORCID: https://orcid.org/0000-0002-9392-9382 \\ Instituto Federal de São Paulo, Brasil \\ E-mail: juliano.massocalegal@gmail.com \\ Thiago Rocha Rodrigues \\ ORCID: https://orcid.org/0000-0002-7472-7929 \\ Universidade Estadual Paulista, Brasil \\ E-mail: t.rodrigues@unesp.br \\ Mario Mollo Neto \\ ORCID: https://orcid.org/0000-0002-8341-4190 \\ Universidade Estadual Paulista, Brasil \\ E-mail: mario.mollo@unesp.br \\ Mariana Matulovic da Silva Rodrigueiro \\ ORCID: https://orcid.org/0000-0001-6626-4621 \\ Universidade Estadual Paulista, Brasil \\ E-mail:mariana.matulovic@unesp.br \\ Kassandra Sussi Mustafé Oliveira \\ ORCID: https://orcid.org/0000-0002-5311-5667 \\ Universidade Estadual Paulista, Brasil \\ E-mail: kassandra.oliveira@unesp.br \\ Paulo Sérgio Barbosa dos Santos \\ ORCID: https://orcid.org/0000-0001-8211-3882 \\ Universidade Estadual Paulista, Brasil \\ E-mail: paulo.sb.santos@unesp.br
}

\begin{abstract}
Resumo
Para compreender a utilização do Arduino e Mit App Inventor na comunicação digital, uma pesquisa acerca dos artigos publicados de 2017 a 2021 foram reunidos através da busca nas bases de dado Scielo, IEEE, Science Direct, Scopus e Web of Science utilizando uma String de busca com intuito de reduzir a abrangência de informações, resultando em 30 artigos iniciais. A partir dos artigos obtidos e o auxílio do software StArt e critérios de seleção previamente definidos, as informações presentes nos artigos foram extraídas e separadas em repartições que representam seus objetivos, metodologias e resultados, além do título e autor deste. Apenas 12 trabalhos foram utilizados no final, se tornando parte da Revisão Sistemática e uma base sólida para o objetivo de controlar um motor produtor de biodiesel a partir da comunicação à distância.
\end{abstract}

Palavras-chave: Arduino; Mit app inventor; Comunicação digital; Revisão sistemática; Internet das coisas.

\begin{abstract}
In order to understand the use of Arduino and Mit App Inventor in digital communication, a survey of articles published from 2017 to 2021 were gathered by searching the Scielo, IEEE, Science Direct, Scopus and Web of Science databases using a search string in order to reduce the scope of information, resulting in 30 initial articles. From the articles obtained and with the help of the StArt software and previously defined selection criteria, the information present in the articles was extracted and separated into breakdowns representing their objectives, methodologies, and results, as well as their title and author. Only 12 papers were used in the end, becoming part of the Systematic Review and a solid base for the objective of controlling a biodiesel engine through remote communication.

Keywords: Arduino; Mit app inventor; Digital communication; Systematic Review; Internet of things.

\section{Resumen}

Para entender el uso de Arduino y Mit App Inventor en la comunicación digital, se recopiló un estudio de los artículos publicados desde 2017 hasta 2021 mediante la búsqueda en las bases de datos Scielo, IEEE, Science Direct, Scopus y Web of Science utilizando una cadena de búsqueda con la intención de reducir el alcance de la información, lo que dio como resultado 30 artículos iniciales. A partir de los artículos obtenidos y con la ayuda del software StArt y los criterios de selección previamente definidos, se extrajo la información presente en los artículos y se separó en desgloses que representan sus objetivos, metodologías y resultados, además del título y el autor de este. Al final sólo se
\end{abstract}


utilizaron 12 trabajos, que pasaron a formar parte de la Revisión Sistemática y a constituir una base sólida para el objetivo de controlar un motor productor de biodiésel desde la comunicación a distancia.

Palabras clave: Arduino; Mit App Inventor; Comunicación digital; Revisión Sistemática; Internet de las cosas.

\section{Introdução}

No mundo atual, é visível que a comunicação digital e a Internet das Coisas (IoT) está cada vez mais predominante, seja em indústrias ou no ambiente doméstico, se tornando cada dia mais o foco de estudos, uma vez que facilita ações humanas através do controle à distância, seja para analisar um resultado fornecido pelo sistema ou interferir diretamente no processo, mudando proporções, um valor o programa ou até mesmo parando o funcionamento através de um simples aparelho telefônico, dado estes exemplos acima, é perceptível a quantidade de usos que a comunicação digital pode fornecer.

Dado isso, é notável que a automação de processos industriais sejam extremamente necessários para a evolução geral da sociedade em que vivemos, por esse motivo que essa Revisão Sistemática da Literatura (RSL) tem como intuito entender o uso do microcontrolador Arduino e da aplicação Mit App inventor, utilizando de dados sólidos obtidos através de artigos recentes relacionados, tornando possível a implementação de ambos, Arduino e Mit App Inventor, em um processo de controle, neste caso, de um reator de biodiesel.

Sabendo da ideia principal de sermos capaz de controlar um reator de biodiesel através da comunicação digital, essa RSL foi feita, buscando entender os usos do Arduino e Mit App Inventor nos últimos 5 anos (desde 2017), tornando os dados encontrados mais confiáveis, tornando possível que o objetivo de controlar os resultados do reator de biodiesel, seu estado, se há problemas em seu funcionamento ou se necessita de manutenção, seja atingido.

Com relação aos Objetivos de Desenvolvimento Sustentável (ODS) da Organização das Nações Unidas (ONU), este método de controle se encaixa nos objetivos 7 e 9, sendo eles, Energia Acessível e Limpa e Indústria, Inovação e Infraestrutura, respectivamente, ambas se encaixam no objetivo do projeto, uma vez que se trata de um projeto na área industrial que tem inovações nos usos de componentes e aplicações que já eram utilizadas com outras finalidades, sendo assim, importante para o desenvolvimento industrial, além de se tratar do controle de um reator biodiesel, que é capaz de produzir energia limpa, atra vés de óleos usados, por exemplo. Buscamos concluir esse projeto fazendo uso do menor número de recursos possíveis, tornando uma opção de controle de baixo custo e alto desempenho, tornando-se um sistema de bom custo-benefício para este reator mencionado anteriormente.

\section{Metodologia}

Dado a complexidade e importância da comunicação digital, a busca por entender como ela funciona e é usada se faz necessária, por este motivo que uma busca foi realizada através da Revisão Sistemática da Literatura (RSL), a qual englobava artigos que se aproximassem do objetivo previamente definido. A RSL é um tipo de estudo que, utilizando de artigos pesquisados, relacionados ao tema do trabalho, seleciona, analisa, avalia e sintetiza estes artigos previamente ditos, com o objetivo de criar um embasamento, que ajuda diretamente no projeto que busca se desenvolvido, além de poder levar dados importantes para pesquisadores futuros.

Inicialmente, para começar a RSL os artigos foram pesquisados em cinco bases de dados diferentes, sendo elas, IEEE, Scielo, Science Direct, Scopus e Web of Science, em todas, apenas artigos que fossem datados a partir de 2017, e que fossem definitivamente, artigos, foram separados e o resultado dessa busca se encontra presente no Gráfico 1. 
Gráfico 1 - Total de trabalhos encontrados nas bases de dados.

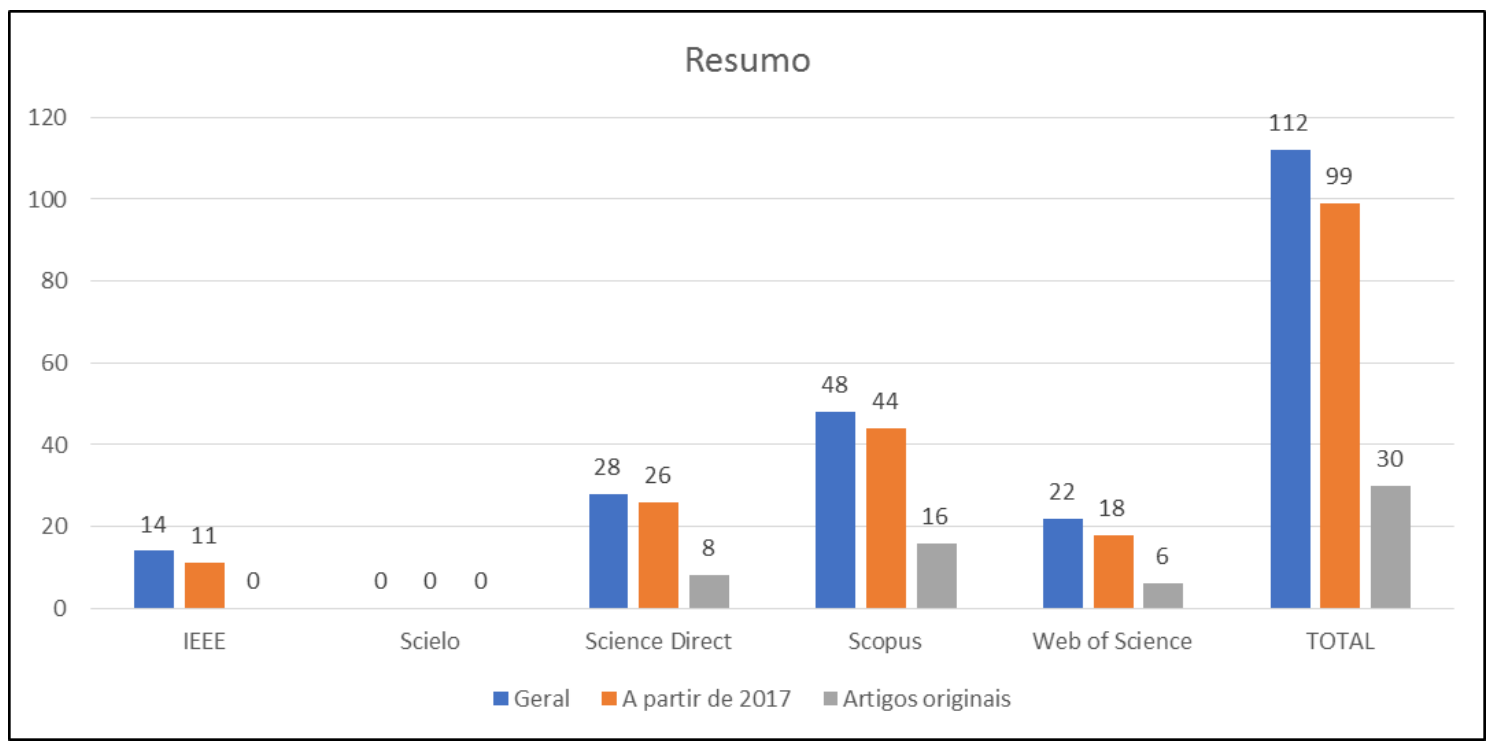

Fonte: Autores (2021).

No gráfico 1, é possível perceber quantos artigos foram obtidos sem exclusão alguma, após selecionar apenas aqueles que foram publicados após o ano de 2017, e posteriormente, apenas os que fossem artigos científicos. Esta exclusão resultou num total de 30 artigos selecionados, os quais serão levados à próxima etapa de exclusão. É importante ressaltar que o número final de trabalhos a partir de 2017 e que fossem artigos foi menor do que o esperado, isso se deve em parte, pela busca nas plataformas Scielo e IEEE, dado em que ambas não foram encontrados artigos que tivessem data de publicação posterior ao ano de 2017.

A etapa seguinte faz uso do software StArt (State of the Art through Systematic Review), uma plataforma que possibilita, através de critérios de exclusão, inclusão, palavras chaves, avaliações e prioridades, selecionar os melhores artigos daqueles que foram previamente obtidos nas bases de dados, separando apenas os que forem relevantes para a pesquisa.

Após adicionar os arquivos, dois processos simples de exclusão acontecem, começando primeiramente pela exclusão de artigos duplicados, seguido da determinação da relevância do trabalho, a partir das palavras-chaves que buscamos e que estão presentes nos títulos, resumos e palavras-chaves dos trabalhos pesquisados nos bancos de dados. As palavras-chaves que mais apareceram frequentemente nos trabalhos foram, em ordem decrescente, Arduino, Mit App Inventor, Arduino Uno, Bluetooth Module e IOT, além de outras que tiveram uma baixa frequência.

\subsection{Entrada}

Para começar, critérios iniciais foram definidos, afunilando o que fosse ser pesquisado, evitando uma pesquisa rasa sobre diversos conteúdos, sabendo disso, um protocolo foi feito a partir de uma base, o qual nos relembra os objetivos iniciais da pesquisa, além de armazenar dados importantes que serão usados futuramente, como os critérios de inclusão e exclusão. Segue o Quadro 1, contendo os parâmetros ditos acima. 
Quadro 1 - Critérios pré-definidos da RSL.

\begin{tabular}{|l}
\hline Objetivo \\
Entender como o conjunto, Arduino e Mit App Inventor, estão sendo utilizados na atualidade \\
Situação problema \\
Realizar uma contextualização sobre usos do Arduino e Mit App Inventor \\
Bases de Dados \\
IEEE, Scielo, Scopus, Science Direct e Web of Science \\
String de busca \\
Arduino AND Mit App Inventor \\
Palavras-Chave \\
Arduino, Arduino UNO, Biodiesel, Control System, IOT, Internet of Things, Mit App Inventor, Smart Control \\
Critérios de inclusão dos artigos nas bases de dados \\
Inclusão (I) - Trabalhos relacionados à Internet das Coisas \\
Inclusão (I) - Trabalhos focados em automação do processo de produção de Biodiesel \\
Inclusão (I) - Trabalhos em Português, Inglês ou Espanhol \\
Inclusão (I) - Trabalhos relacionados à comunicação digital \\
Critérios de exclusão dos artigos nas bases de dados \\
Exclusão (E) - Trabalhos em outros idiomas que não sejam Português, Inglês e Espanhol \\
Exclusão (E) - Trabalhos que não abordem sobre comunicação digital \\
Exclusão (E) - Trabalho que abordem muito pouco sobre comunicação digital \\
\hline
\end{tabular}

Fonte: Autores (2021).

Seguido desse gráfico, a fase de processamento se inicia, utilizando esses mesmos dados como critérios para inclusão e exclusão de trabalhos que se aproximem ou se distanciem da busca inicial, respectivamente.

\subsection{Processamento}

Utilizando o software, os critérios foram utilizados, fazendo uma exclusão nos artigos que não fossem relevantes para a pesquisa. Como visto no gráfico 1, tínhamos inicialmente 30 artigos que atendessem nossa busca, desses, apenas 14 passaram pela etapa de seleção, é possível ver uma representação dessa exclusão no Gráfico 2.

Gráfico 2 - Relação de artigos aceitos, rejeitados e duplicados no processo de seleção.

\section{Status}

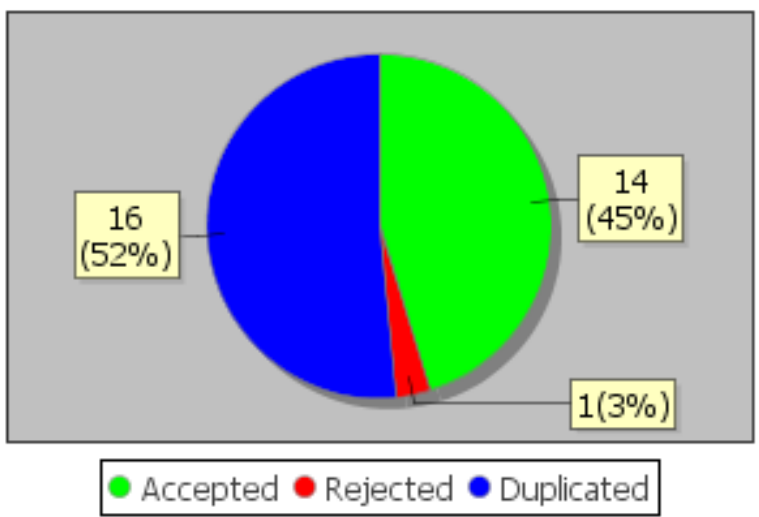

Fonte: Autores (2021). 
Todos os 14 trabalhos que foram selecionados passaram pela etapa de extração, na qual analisamos cuidadosamente os resumos destes trabalhos que passaram pela etapa de seleção, determinando quais são relevantes, através dos métodos utilizados, explicações, se possuem desenvolvimento e resultados importantes para a pesquisa. O número de trabalhos que conseguiram passar pelo processo de extração podem ser vistos no Gráfico 3.

Gráfico 3 - Relação de trabalhos aceitos e rejeitados no processo de extração.

\section{Status}

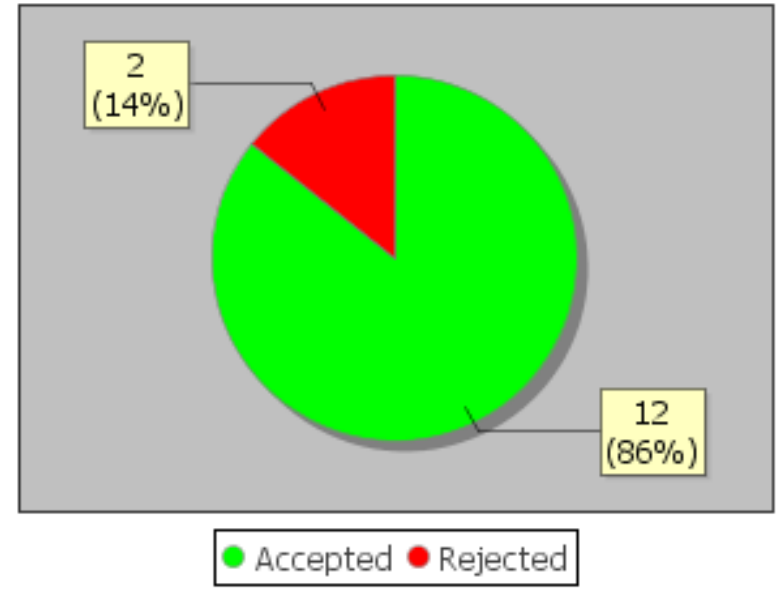

Fonte: Autores (2021).

Dos 14 trabalhos que chegaram à etapa de extração, 12 deles conseguiram atender aos requisitos, todos estes provando possuir conteúdo o suficiente para serem relevantes para o que buscamos atingir através da comunicação digital.

\section{Resultados e Discussão}

Depois de todo o processo de seleção e exclusão utilizando dos critérios de inclusão e exclusão presentes no Quatro 1, foi possível escolher os 12 melhores que se sobressaíram sobre os outros, apresentando informações e objetivos mais relevantes para o projeto.

Uma nuvem de palavras baseada nas palavras-chaves mais encontradas nos trabalhos que foram extraídos foi feita através do software StArt, nela, as palavras mais presentes são Arduino, Bluetooth Module, Bluetooth, IOT e Mit App Inventor 2, além de outras palavras que aparecem menos frequentemente como Android, Arduino Uno, a Figura 1 ilustra a nuvem de palavras.

Desses 12 que foram selecionados, um resumo o qual apresentará os autores destes, o ano de publicação, título, metodologia e resultados obtidos será mostrado a seguir em forma de quadro, facilitando o entendimento e se adequando aos padrões. Essas informações podem ser analisadas no Quadro 2. 
Figura 1 - Nuvem de palavras com as palavras mais encontradas nas palavras chaves dos artigos.

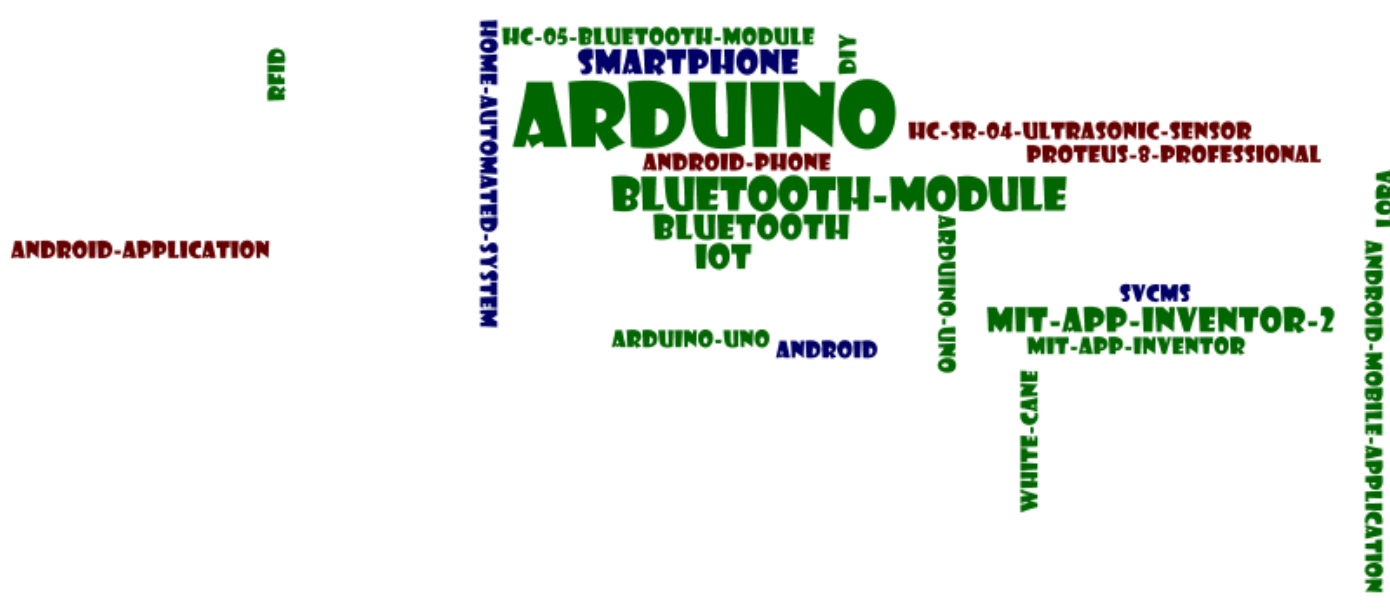

Fonte: Autores (2021). 
Quadro 2 - Sumarização dos artigos que foram extraídos.

\begin{tabular}{|c|c|c|c|c|c|}
\hline TITULO & AUTOR & ANO & OBJETIVOS & METODOLOGIA & RESULTADOS \\
\hline $\begin{array}{l}\text { An Intelligent Walking Stick for } \\
\text { the Visually-Impaired People }\end{array}$ & $\begin{array}{c}\text { Nowshin, Nadia and } \\
\text { Shadman, Sakib and Joy, } \\
\text { Saha and Aninda, Sarker } \\
\text { andMinhajul, Islam Md }\end{array}$ & 2017 & $\begin{array}{l}\text { O objetivo inicialmente pro- } \\
\text { posto foi de projetar uma ben- } \\
\text { gala inteligente que seria capaz } \\
\text { de localizar objetos e líquidos } \\
\text { e avisar o deficiente visual. }\end{array}$ & $\begin{array}{l}\text { Foi feito uso da plataforma Mit App Inventor, } \\
\text { do microcontrolador Arduino Nano e de uma } \\
\text { série de outros componentes, como um sensor } \\
\text { ultrassônico HC SR-04, um módulo Bluetooth, } \\
\text { um capacitor de cerâmica, botões de pressão, } \\
\text { uma bateria de } 9 \mathrm{~V} \text { e um buzzer. Quando em } \\
\text { conjunto, o sensor irá detectar presença de ob- } \\
\text { jetos físicos ou líquidos numa certa distância, } \\
\text { fazendo com que um som seja produzido, avi- } \\
\text { sando para a pessoa incapaz de ver que ela deve } \\
\text { ser cautelosa. }\end{array}$ & $\begin{array}{l}\text { O resultado foi extremamente significante, pos- } \\
\text { suindo uma alta precisão, quase atingindo a per- } \\
\text { feição, é possível utilizar a bengala inteligente } \\
\text { de olhos fechados sem maiores problemas além } \\
\text { de que não é um conjunto de componentes ca- } \\
\text { ros, podendo ser feito por um baixo valor. A de- } \\
\text { tecção de obstáculos próximos atingiu um su- } \\
\text { cesso de } 90 \% \text {. }\end{array}$ \\
\hline $\begin{array}{l}\text { A speech activated control sys- } \\
\text { tem for infrared appliances }\end{array}$ & $\begin{array}{l}\text { Chigozirim, A. and Olu- } \\
\text { watofunmi, A. and } \\
\text { Nwaocha, V.O. and Juli- } \\
\text { ana, N. }\end{array}$ & 2020 & $\begin{array}{l}\text { Produzir um suporte capaz de } \\
\text { permitir antigos dispositivos } \\
\text { infravermelhos aproveitarem } \\
\text { de comandos de voz. }\end{array}$ & $\begin{array}{l}\text { Foi realizado o projeto utilizando da linguagem } \\
\text { de programação Arduino, Java e Mit App In- } \\
\text { ventor, além de um Arduino Nano, um módulo } \\
\text { bluetooth e um transmissor IR na parte física, } \\
\text { todos foram utilizados para produzir o suporte. }\end{array}$ & $\begin{array}{l}\text { O protótipo realizado durante o estudo se pro- } \\
\text { vou eficiente o suficiente para ser fácil de utili- } \\
\text { zar e entender seu funcionamento, uma vez que } \\
\text { era um suporte. Sua funcionalidade é útil, per- } \\
\text { mitindo que funções que antes eram impossí- } \\
\text { veis sem o controle de voz, agora sejam possí- } \\
\text { veis através desse suporte. }\end{array}$ \\
\hline $\begin{array}{l}\text { Developing an electrical outlet } \\
\text { using internet of things (Iot) }\end{array}$ & Arispe, M.C.A. et al. & 2020 & $\begin{array}{l}\text { O objetivo inicial era produzir } \\
\text { um protótipo capaz de testar o } \\
\text { tempo de ativação e resposta } \\
\text { entre a tomada elétrica desen- } \\
\text { volvida através da Internet das } \\
\text { Coisas e uma comum. }\end{array}$ & $\begin{array}{l}\text { Foi utilizado o microcontrolador NodeMCU e } \\
\text { internet, com base na lógica da internet das coi- } \\
\text { sas para procurar entender algumas concepções } \\
\text { e testar diferentes reações e tempo de reação de- } \\
\text { pendendo de como a tomada fosse ligada. }\end{array}$ & $\begin{array}{l}\text { O projeto podia ser acessado de qualquer dis- } \\
\text { tância de forma remota. O resultado mostrou } \\
\text { que a internet foi capaz de acelerar o tempo de } \\
\text { ativação, porém não houve grandes diferenças } \\
\text { no tempo de resposta de ativação, cumprindo, } \\
\text { no geral, o objetivo inicial. }\end{array}$ \\
\hline $\begin{array}{c}\text { A cloud-based smart parking } \\
\text { system based on IoT Technolo- } \\
\text { gies }\end{array}$ & $\begin{array}{c}\text { Mukadam, Z. e Logeswa- } \\
\text { ran, R. }\end{array}$ & 2020 & $\begin{array}{l}\text { Um sistema de estaciona- } \\
\text { mento, capaz de entender atra- } \\
\text { vés de sensores se vagas já es- } \\
\text { tavam ocupadas, era o objetivo } \\
\text { inicial. }\end{array}$ & $\begin{array}{l}\text { Utilizando Arduino UNOs, Node MCUs, Ras- } \\
\text { pberry Pi } 3 \text { e o Mit App Inventor } 2 \text {, um sistema } \\
\text { foi projetado utilizando como base a Internet } \\
\text { das Coisas, enviando os dados armazenados } \\
\text { para uma nuvem de dados. Além disso, um sis- } \\
\text { tema capaz de detectar entrada e saída de veícu- } \\
\text { los, placas e rejeição de veículos grandes tam- } \\
\text { bém foi adicionado ao circuito original. }\end{array}$ & $\begin{array}{l}\text { O protótipo foi um sucesso, apresentando uma } \\
\text { grande taxa de acerto com um pequeno tempo } \\
\text { de reação e quase nenhum problema, podendo } \\
\text { ser considerado extremamente funcional, além } \\
\text { disso, obteve uma nota de retorno de } 4.2 \text { de } 5 \text { e } \\
\text { uma preferência de } 90 \% \text { dos usuários deste sis- } \\
\text { tema. }\end{array}$ \\
\hline
\end{tabular}




\begin{tabular}{|c|c|c|c|c|c|}
\hline $\begin{array}{l}\text { Weather prediction using multi- } \\
\text { ple iot based wireless sensors }\end{array}$ & Sah, H.K. e Koli, S.M. & 2019 & $\begin{array}{l}\text { Foi iniciado um projeto de um } \\
\text { aplicativo capaz de fazer previ- } \\
\text { sões meteorológicas a partir de } \\
\text { sensores sem fios. }\end{array}$ & $\begin{array}{l}\text { Mais de um tipo de sistema foi desenvolvido, os } \\
\text { componentes utilizados foram o módulo Node- } \\
\text { MCU Wifi ou o microcontrolador Arduino, co- } \\
\text { nexão de internet, base de dados na nuvem, tec- } \\
\text { nologia UDP, HTTP e Bluetooth. Para a criação } \\
\text { de um aplicativo onde os dados seriam mostra- } \\
\text { dos, o Mit App Inventor foi utilizado com obje- } \\
\text { tivo de criar essa interface que apresenta os va- } \\
\text { lores de umidade, temperatura, chuva em tempo } \\
\text { real e nível de monóxido de carbono. }\end{array}$ & $\begin{array}{l}\text { Este projeto, realizado com diversos tipos de } \\
\text { componentes e tecnologias diferentes se apre- } \\
\text { sentou extremamente promissor, podendo ser } \\
\text { utilizado num futuro para desenvolver ainda } \\
\text { mais o ramo de previsão meteorológica. Os re- } \\
\text { sultados foram bons, além de que as previsões } \\
\text { eram corretas na maior parte. }\end{array}$ \\
\hline $\begin{array}{l}\text { A Smart Voltage and Current } \\
\text { Monitoring System for Three } \\
\text { Phase Inverters Using an An- } \\
\text { droid Smartphone Application }\end{array}$ & $\begin{array}{l}\text { Mnati, Mohannad Jabbar } \\
\text { et al. }\end{array}$ & 2017 & $\begin{array}{l}\text { Foi planejado um sistema de } \\
\text { monitoração de tensão e cor- } \\
\text { rente para um sistema elétrico } \\
\text { de três fases utilizando Ar- } \\
\text { duino e aplicativo de celular. }\end{array}$ & $\begin{array}{l}\text { O Arduino e o Mit App Inventor foram utiliza- } \\
\text { dos. A plataforma Arduino é responsável por } \\
\text { enviar os valores calculados através de um Blu- } \\
\text { etooth HC-05, para um aplicativo feito pelo Mit } \\
\text { App Inventor, responsável por mostrar os valo- } \\
\text { res e monitorar algumas propriedades funda- } \\
\text { mentais de qualidade da tensão. }\end{array}$ & $\begin{array}{l}\text { Este sistema de monitoração tem um melhor } \\
\text { custo benefício que modelos similares além de } \\
\text { ser mais seguro que medir as tensões da rede } \\
\text { frequentemente. O sistema de monitoramento } \\
\text { foi um sucesso, sendo um sistema de baixo } \\
\text { custo e facilmente aplicável. }\end{array}$ \\
\hline $\begin{array}{l}\text { Design and Implementation of } \\
\text { an Intelligent Windowsill Sys- } \\
\text { tem Using Smart Handheld De- } \\
\text { vice and Fuzzy Microcontroller }\end{array}$ & $\begin{array}{l}\text { Wang, Jing-Min; Yang, } \\
\text { Ming-Ta e Chen, Po-Lin }\end{array}$ & 2017 & $\begin{array}{l}\text { Um peitoril de janela inteli- } \\
\text { gente que é capaz de medir a } \\
\text { iluminação interna, a tempera- } \\
\text { tura, umidade, concentração } \\
\text { de gás carbônico e a direção } \\
\text { da chuva e vento da área ex- } \\
\text { terna foram desenvolvidos. }\end{array}$ & $\begin{array}{l}\text { Os valores de temperatura, iluminação interna, } \\
\text { umidade e concentração de gás carbônico são } \\
\text { medidos através de sensores, que são enviados } \\
\text { para um Arduino, dependendo desses valores, } \\
\text { um sinal será enviado para controlar a cortina } \\
\text { e janela, ambas elétricas, para que se adaptem } \\
\text { as condições do ambiente. }\end{array}$ & $\begin{array}{l}\text { Esse projeto cumpriu seu papel no quesito con- } \\
\text { veniência para quem fazer uso do mesmo, já } \\
\text { que não seria necessário se preocupar com as } \\
\text { janelas da casa em um tempo de chuva, por } \\
\text { exemplo. Além disso, ele provou ter uma boa } \\
\text { taxa de acerto em testes. }\end{array}$ \\
\hline $\begin{array}{l}\text { Design and implementation of a } \\
\text { smart monitoring system for } \\
\text { water quality of fish farms }\end{array}$ & $\begin{array}{l}\text { Salih, N.A.J.; Hasan, I.J. } \\
\text { e Abdulkhaleq, N.I. }\end{array}$ & 2019 & $\begin{array}{l}\text { Foi planejado um sistema de } \\
\text { monitoramento de uma pisci- } \\
\text { cultura capaz de informar a } \\
\text { qualidade da água (pH) e a } \\
\text { temperatura da água. }\end{array}$ & $\begin{array}{l}\text { Este sistema que foi planejado era divido em } \\
\text { duas partes, uma de medição e outra de monito- } \\
\text { ramento. Na parte de medição foi utilizado um } \\
\text { Arduino UNO para medir os valores de pH e } \\
\text { temperatura recebido pelos sensores. As infor- } \\
\text { mações são enviadas através de Bluetooth para } \\
\text { a segunda parte. Essa segunda parte é onde há a } \\
\text { monitoração do estado de todo o sistema através } \\
\text { de um aplicativo feito utilizando Mit App In- } \\
\text { ventor 2 } \\
\end{array}$ & $\begin{array}{l}\text { Esse projeto desenvolvido se provou útil, já que } \\
\text { é capaz de monitorar as pisciculturas a longas } \\
\text { distâncias com um baixo custo e alta confiabili- } \\
\text { dade. Os testes se provaram um sucesso, tendo } \\
\text { alta precisão e uma interface que era facilmente } \\
\text { capaz de ser analisada. }\end{array}$ \\
\hline $\begin{array}{l}\text { Vehicle security system using } \\
\text { short message service (SMS) as } \\
\text { a danger warning in motorcycle } \\
\text { vehicles }\end{array}$ & Astuti, N.P. et al. & 2020 & $\begin{array}{l}\text { Um sistema que irá avisar o } \\
\text { usuário do aplicativo através } \\
\text { de uma mensagem SMS e en- } \\
\text { viar as coordenadas atuais do } \\
\text { veículo da pessoa caso o veí- } \\
\text { culo estiver em perigo. }\end{array}$ & $\begin{array}{l}\text { Para a realização desse projeto um Arduino } \\
\text { Atmega foi utilizado, além de um módulo de } \\
\text { GPS neo 6, um módulo SIM GSM 800L, um } \\
\text { celular, aplicação Google Maps e um software } \\
\text { que fazia uso da linguagem C e do aplicativo } \\
\text { Mit App Inventor. }\end{array}$ & $\begin{array}{l}\text { O projeto cumpriu seu papel, e era capaz de mo- } \\
\text { nitorar bem a localização de um veículo. Al- } \\
\text { guns ajustes tiveram que ser feitos para que o } \\
\text { projeto funcionasse, sem alterar o projeto ao } \\
\text { todo, apenas simples mudanças. }\end{array}$ \\
\hline
\end{tabular}




\begin{tabular}{|c|c|c|c|c|c|}
\hline $\begin{array}{l}\text { Smart prepaid traffic fines sys- } \\
\text { tem using RFID, IoT and mo- } \\
\text { bile app }\end{array}$ & Al-abassi, S.A.W. et al. & 2019 & $\begin{array}{l}\text { Foi projetado um sistema ca- } \\
\text { paz de auxiliar oficiais de trân- } \\
\text { sito a realizar seu serviço com } \\
\text { o uso de um aplicativo. }\end{array}$ & $\begin{array}{l}\text { Componentes como um identificador de radio- } \\
\text { frequência (RFID), um tag passivo, uma uni- } \\
\text { dade de processamento foi utilizada no desen- } \\
\text { volvimento do projeto, além de um microcon- } \\
\text { trolador Arduino. O RFID coletava os dados } \\
\text { que eram enviados através da internet para um } \\
\text { servidor que processa os dados através de regras } \\
\text { pré-determinadas, retornando ao usuário atra- } \\
\text { vés de uma interface desenvolvida através do } \\
\text { Mit App Inventor. } \\
\end{array}$ & $\begin{array}{l}\text { O protótipo teve sucesso e conseguiu funcionar } \\
\text { extremamente bem, funcionando da forma es- } \\
\text { perada no início do projeto, conseguindo uma } \\
\text { alta taxa de precisão. O aplicativo também per- } \\
\text { mite monitorações de certos carros e adicionar } \\
\text { novos Ids. }\end{array}$ \\
\hline $\begin{array}{c}\text { Experimental Verification Of } \\
\text { Three Mode Controller For } \\
\text { Home Appliance Using Wire- } \\
\text { less Technology }\end{array}$ & $\begin{array}{l}\text { Wahidh, M.R.A. e Masi- } \\
\text { lamani, M. }\end{array}$ & 2021 & $\begin{array}{l}\text { O objetivo inicial era criar um } \\
\text { sistema de três modos diferen- } \\
\text { tes capaz de controlar uma to- } \\
\text { mada elétrica. }\end{array}$ & $\begin{array}{l}\text { Para realizar o projeto os seguintes componen- } \\
\text { tes foram utilizados: Microcontrolador de baixo } \\
\text { custo (Arduino), um módulo Bluetooth e um } \\
\text { aplicativo Android. Os três modos desenvolvi- } \\
\text { dos foram: modo de mudança manual, modo } \\
\text { utilizando resistor do tipo LDR e modo baseado } \\
\text { na comunicação sem fio Bluetooth. }\end{array}$ & $\begin{array}{l}\text { Todos os três tipos de modos foram projetados. } \\
\text { Todos eles, sem exceção, conseguiram uma } \\
\text { precisão de } 100 \% \text {, um resultado extremamente } \\
\text { relevante para o objetivo inicial, sendo um pro- } \\
\text { jeto com um alto grau de sucesso. }\end{array}$ \\
\hline $\begin{array}{l}\text { Mobile technology system ffor } \\
\text { health intervention (M-health) }\end{array}$ & Jain, A. et al. & 2019 & $\begin{array}{l}\text { O objetivo inicial era um pro- } \\
\text { jeto que permitiria monitorar } \\
\text { os batimentos cardíacos e tem- } \\
\text { peratura corporal de um indiví- } \\
\text { duo. }\end{array}$ & $\begin{array}{l}\text { Para pôr em prática, um microcontrolador Ar- } \\
\text { duino foi utilizado, uma base de celular An- } \\
\text { droid para estabelecer um módulo de tempera- } \\
\text { tura e de batimento cardíaco. O sensor de pulso } \\
\text { AD8232 Single Lead Heart Rate Monitor e o } \\
\text { sensor de temperatura LM35 foram utilizados. } \\
\text { Os valores obtidos são passados para um apli- } \\
\text { cativo feito no Mit App Inventor } 2 \text { através de } \\
\text { um sistema Bluetooth. Os dados recebidos po- } \\
\text { dem ser analisados para saber os batimentos por } \\
\text { minuto da pessoa, sua temperatura corporal em } \\
\text { graus Celsius e um Eletrocardiograma. }\end{array}$ & $\begin{array}{l}\text { O projeto atingiu parcialmente seu objetivo e os } \\
\text { criadores dizem que com equipamentos melho- } \\
\text { res seria possível obter um melhor resultado, } \\
\text { podendo obter de melhor forma os valores, } \\
\text { além de processar e entregar da melhor forma } \\
\text { possível. }\end{array}$ \\
\hline
\end{tabular}

Fonte: Autores. 


\section{Conclusão}

Após o estudo desses trabalhos, foi possível perceber que especificamente, a utilização do microcontrolador Arduino e o aplicação Mit App Inventor, não são muito comuns de serem usados em conjunto para realizar projetos, uma vez que o número de trabalhos relacionados se provou relativamente baixo, entretanto, os trabalhos extraídos possuem informações importantes que serão essenciais no decorrer do projeto.

Sobre o assunto principal presente nos artigos selecionados, a comunicação digital se provou o principal, estando presente de forma nítida ou mais implícita em todos os artigos, dessa forma é possível criar uma ligação mental entre o uso do Arduino e Mit App Inventor e a comunicação digital, uma vez que é exatamente isso que ambos proporcionam quando utilizados em conjunto. As informações extraídas descreveram os diversos processos utilizados para que essa comunicação usuário-máquina ocorresse, atingindo um dos objetivos inicialmente planejados para a RSL.

Essa RSL que foi realizada se mostra uma possível base de estudo, dado que descreve os estudos realizados nos anos determinados inicialmente pela pesquisa, sendo de utilidade para pesquisadores com objetivos de desenvolver projetos que se assemelhem a área desta RSL.

\section{Referências}

Al-abassi, S. A. W. et al (2019, August). Smart prepaid traffic fines system using RFID, IoT and mobile app. Telkomnika (Telecommunication Computing Electronics and Control), 17(4), 1828-37. https://doi.org/doi.org/10.12928/TELKOMNIKA.v17i4.10771.

Arispe, M. C. A. et al (2020). Developing an electrical outlet using internet of things (Iot). International Journal of Advanced Trends in Computer Science and Engineering, 9(1.3), 280-86. https://doi.org/doi.org/10.30534/ijatcse/2020/4291.32020.

Astuti, N. P. et al (2020). Vehicle security system using short message service (SMS) as a danger warning in motorcycle vehicles. Journal of Robotics and Control (JRC), 1(6), 224-28. https://doi.org/doi.org/10.18196/jrc.1642.

Chigozirim, A. et al (2020, February 29). A speech activated control system for infrared appliances. Journal Europeen des Systemes Automatises, 53(1), 103-10. https://doi.org/doi.org/10.18280/jesa.530113.

Conforto, E. C., Amaral, D. C., \& Silva, S. L. d. (2011). Roteiro para revisão bibliográfica sistemática: aplicação no desenvolvimento de produtos e gerenciamento de projetos. $8^{\circ}$ CONGRESSO BRASILEIRO DE GESTÃO DE DESENVOLVIMENTO DE PRODUTO - CBGDP 2011, 1-12

Jain, A. et al (2019, April). Mobile technology system ffor health intervention (M-health). International Journal of Innovative Technology and Exploring Engineering, 8(6S4), 977-81. https://doi.org/doi.org/10.35940/ijitee.F1200.0486S419.

Mnati, M. J. et al. (2017, April 15). A Smart Voltage and Current Monitoring System for Three Phase InvertersUsing an Android Smartphone Application. SENSORS, 17(4). https://doi.org/doi.org/10.3390/s17040872.

Mukadam, Z., \& Logeswaran, R. (2020). A cloud-based smart parking system based on IoT technologies. Journal of Critical Reviews. https://doi.org/doi.org/10.31838/jcr.07.03.20.

Nowshin, N. et al (2017, November 22). An Intelligent Walking Stick for the Visually-Impaired People. INTERNATIONAL JOURNAL OF ONLINE ENGINEERING, 13(11), 94-101. https://doi.org/http://doi.org/10.3991/ijoe.v13i11.7565.

Sah, H. K., \& Koli, S. M. (2019, October 30). Weather prediction using multiple iot based wireless sensors. International Journal of Engineering and Advanced Technology, 9(1), 3995-9. https://doi.org/doi.org/10.35940/ijeat.A1037.109119.

Salih, N. A. J. et al (2019, April). Design and implementation of a smart monitoring system for water quality of fish farms. Indonesian Journal of Electrical Engineering and Computer Science, 14(1), 44-50. https://doi.org/doi.org/10.11591/ijeecs.v14.i1.pp44-50.

Wahidh, M. R. A., \& Masilamani, M. (2021, July 4). Experimental Verification Of Three Mode Controller For Home Appliance Using Wireless Technology. IIUM Engineering Journal, 22(2), 188-98. https://doi.org/doi.org/10.31436/iiumej.v22i2.1618.

Wang, J. et al (2017, April 11). Design and Implementation of an Intelligent Windowsill System UsingSmart Handheld Device and Fuzzy Microcontroller. SENSORS, 17(4). https://doi.org/doi.org/10.3390/s17040830. 